\title{
Clinical efficacy and safety of statins in managing cardiovascular risk
}

\author{
Navin K Kapur' \\ Kiran Musunuru² \\ 'Division of Cardiology, Tufts \\ University - New England Medical \\ Center; Boston, MA, USA; ${ }^{2}$ Division \\ of Cardiology, Johns Hopkins \\ University School of Medicine, \\ Baltimore, MD, USA
}

\begin{abstract}
Since their introduction in the 1980s, 3-hydroxy-3-methylglutaryl coenzyme A (HMG-CoA) reductase inhibitors (statins) have emerged as the one of the best-selling medication classes to date, with numerous trials demonstrating powerful efficacy in preventing cardiovascular outcomes. As our understanding of low-density lipoprotein cholesterol (LDL-C) and atherosclerosis continues to grow, the concept of 'lower is better' has corresponded with a 'more is better' approach to statin-based therapy. This review provides a detailed understanding of the clinical efficacy and safety of statins with a particular emphasis on the third generation drug, rosuvastatin.
\end{abstract}

Keywords: low density lipoprotein cholesterol (LDL-C), efficacy, safety, HMGCo-A reductase inhibitors, rosuvastatin

\section{Introduction}

In 1976, the Japanese scientist Akira Endo identified a fungal metabolite that blocks cholesterol synthesis by inhibiting the enzyme 3-hydroxy-3-methylglutaryl coenzyme A (HMG-CoA) reductase, resulting in the first 'statin' agent, mevastatin (Endo 2004). In addition to blocking this key enzyme involved in cholesterol synthesis, statins possess numerous pleiotropic properties including: (i) nitric oxide-mediated improvement of endothelial dysfunction and upregulation of endothelin-1 expression; (ii) antioxidant effects; (iii) anti-inflammatory properties; (iv) inhibition of cell proliferation with anticarcinogenic actions in animals; (v) stabilization of atherosclerotic plaques; (vi) anticoagulant effects; and (vii) inhibition of graft rejection after heart and kidney transplantation (Davignon and Laaksonen 1999). While the role of statin pleiotropic effects in cardiovascular prevention remains to be determined, statins have become one of the best-selling medication classes to date since their introduction into the marketplace in 1986, and include the following drugs commercially available in the US (in order of introduction): lovastatin, pravastatin, fluvastatin, atorvastatin, simvastatin, and rosuvastatin.

Since the first Adult Treatment Panel (ATP) recommendations in 1988 (Expert Panel on Detection, Evaluation, and Treatment of High Blood Cholesterol in Adults [Adult Treatment Panel II] 1993), published guidelines have focused on aggressive management of elevated low density lipoprotein cholesterol (LDL-C) in populations at risk for coronary heart disease (CHD). According to the National Cholesterol Education Panel (NCEP) ATP III guidelines published in 2001, patients with established CHD, non-coronary atherosclerosis, diabetes mellitus, or greater than two major cardiac risk factors with a calculated Framingham risk score of greater than $20 \%$ are candidates for the most aggressive LDL-C goal of less than $100 \mathrm{mg} / \mathrm{dL}$ (Expert Panel on Detection, Evaluation, and Treatment of High Blood Cholesterol in Adults [Adult Treatment Panel III] 2001). Subsequently, the Heart Protection Study 
(HPS) (Heart Protection Study Collaborative Group 2003) and Pravastatin or Atorvastatin Evaluation and Infection Therapy (PROVE-IT) (Cannon et al 2004) trial reported incremental $22 \%$ and $16 \%$ reductions in risk of $\mathrm{CHD}$ events with LDL-C levels lowered below $100 \mathrm{mg} / \mathrm{dL}$ in high-risk patients. As a result, major updates were proposed to the 2001 guidelines. An 'optional' target of LDL-C less than $70 \mathrm{mg} / \mathrm{dL}$ was recommended for patients at 'very high risk' of coronary events, which includes established CHD plus either non-coronary atherosclerosis, diabetes mellitus, or a greater than 20\% calculated Framingham 10-year CHD risk. Furthermore, individuals with two or more risk factors and a calculated Framingham 10-year CHD risk of 10\%-20\% have the 'optional' target LDL-C of $<100 \mathrm{mg} / \mathrm{dL}$ (Grundy et al 2004). While statins have become first-line pharmacological therapy to attain the LDL-C goals laid out by the NCEP/ATP III guidelines, the clinical efficacy of each drug varies significantly (Table 1). This review summarizes the clinical efficacy of the various statins with a particular focus on rosuvastatin.

\section{First generation statins}

Lovastatin, pravastatin, and fluvastatin were introduced in the US in the late 1980s and 1990s; they represent the class members with the lowest potency. Typically, these statins need to be taken in doses of 40-80 mg daily to exceed a $30 \%$ reduction in LDL cholesterol levels (Jones et al 1998). Of the three, pravastatin has been most rigorously tested in controlled clinical trials and has demonstrated improved cardiovascular outcomes. Three trials - the West of Scotland Coronary Prevention Study (WOSCOPS), the Prospective Study of Pravastatin in the Elderly at Risk (PROSPER), and the Antihypertensive and Lipid-Lowering Treatment to Prevent Heart Attack Trial (ALLHAT-LLT) - evaluated the use of pravastatin at the dose of $40 \mathrm{mg}$ daily vs no statin therapy in primary prevention patients (ie, those without known CHD). Two of the trials demonstrated reductions in LDL-C levels, cardiac mortality, and coronary events with pravastatin compared to placebo - WOSCOPS with $26 \%$, $28 \%$, and $22 \%$ reductions, respectively, in men (Shepherd et al 1995); and PROSPER with 34\%, 24\%, and 19\% reductions, respectively, in elderly patients (Shepherd et al 2002). Although ALLHAT-LLT patients on pravastatin experienced an LDL-C reduction of $28 \%$, the control group receiving usual care had a reduction of $11 \%$, and no statistically significant differences in coronary events or mortality were seen, perhaps due to the smaller difference in LDL-C levels. The relative lack of benefit in ALLHAT led the NCEP ATP recommendation to lower LDL at least 30\%-40\% for CVD risk reduction (ALLHAT 2002).

Pravastatin has also proved to be effective in secondary prevention patients (ie, patients with a history of myocardial infarction (MI) or symptomatic coronary artery disease). In the Cholesterol And Recurrent Events (CARE) trial, patients receiving $40 \mathrm{mg}$ of pravastatin daily enjoyed a $24 \%$ reduction in coronary events and a non-significant trend towards lower cardiac mortality compared to patients receiving placebo therapy (Sacks et al 1996). Similarly, in the Long-term Intervention with Pravastatin in Ischaemic Disease (LIPID) study, patients on $40 \mathrm{mg}$ of pravastatin daily experienced a $24 \%$ reduction in coronary events, as well as a $24 \%$ reduction in cardiac mortality, compared to control patients (LIPID 1998).

There is less extensive data showing cardiovascular benefits with lovastatin and fluvastatin, although it is presumed that they improve outcomes similarly to pravastatin if titrated to equivalent lipid-lowering effects. Fluvastatin therapy reduces coronary events in special populations of secondary prevention patients including those who have already undergone percutaneous coronary intervention (Serruys et al 2002) and those who have received renal transplants (Holdaas et al 2003). Lovastatin, in contrast, has been assessed in a sizeable primary prevention population in the Air Force/Texas Coronary Atherosclerosis Prevention Study (AFCAPS/TexCAPS), in which men and women with average cholesterol levels receiving 20-40 mg of lovastatin daily saw a mean LDL-C reduction of $25 \%$ and a $37 \%$ reduction in first coronary events (Downs et al 1998).

One particular advantage of pravastatin and fluvastatin is their lower potential for drug - drug interactions, as they are not metabolized by the cytochrome P450 3A4 complex (CYP 3A4) as are many of the other statins; other medications

Table I Lipid-lowering effects of the three generations of statins

\begin{tabular}{|c|c|c|c|c|c|}
\hline Generation & Statins & Change in LDL & Change in HDL & $\begin{array}{l}\text { Change in total } \\
\text { cholesterol }\end{array}$ & Change in triglycerides \\
\hline Ist & Lovastatin, pravastatin, fluvastatin & $-21 \%$ to $-42 \%$ & $+2 \%$ to $+12 \%$ & $-16 \%$ to $-34 \%$ & $-6 \%$ to $-27 \%$ \\
\hline 2nd & Simvastatin, atorvastatin & $-26 \%$ to $-60 \%$ & $+5 \%$ to $+16 \%$ & $-19 \%$ to $-45 \%$ & $-12 \%$ to $-53 \%$ \\
\hline $3 r d$ & Rosuvastatin & $-45 \%$ to $-63 \%$ & $+8 \%$ to $+14 \%$ & $-33 \%$ to $-46 \%$ & $-10 \%$ to $-35 \%$ \\
\hline
\end{tabular}

Source: Adapted from, Vaughan CJ, Gotto AM Jr. 2004. Update on statins: 2003. Circulation, I 10:886-92. 
and foods that modulate CYP 3A4 function will not affect the two statins' activity. Whereas fluvastatin is processed by another P450 complex, pravastatin does not undergo any processing by the cytochrome $\mathrm{P} 450$ system. As such, despite their lower potency they are attractive candidates for use in patients who have proven intolerant of more potent statins such as atorvastatin, simvastatin, or rosuvastatin.

\section{Second generation statins}

The second generation of statins, comprising atorvastatin and simvastatin, are currently the best-selling statins in the US. They have significantly improved efficacy in reducing LDL-C levels compared to the earlier statins. For example, to achieve greater than $30 \%$ reduction in LDL-C levels typically requires $20 \mathrm{mg}$ daily of simvastatin and only $10 \mathrm{mg}$ daily of atorvastatin (Jones et al 1998). A large body of data supports their use in both the primary and the secondary prevention populations. In the earliest trial, the Scandinavian Simvastatin Survival Study (4S), secondary prevention patients receiving 20-40 mg of simvastatin experienced a 35\% mean reduction of LDL-C levels, a $42 \%$ reduction in cardiac mortality, and a $34 \%$ reduction in coronary events compared to patients receiving placebo therapy (Scandinavian Simvastatin Survival Study Group 1994). The finding of benefits with simvastatin therapy was extended to primary prevention patients in the Heart Protection Study (HPS), a large trial with more than 20,000 patients that documented an $18 \%$ reduction in cardiac mortality and $26 \%$ reduction in coronary events with the use of $40 \mathrm{mg}$ of simvastatin daily (Heart Protection Study Collaborative Group 2003).

The first two prospective controlled trials to establish the efficacy of atorvastatin in improving outcomes in primary prevention patients were the Anglo-Scandinavian Cardiac Outcomes Trial-Lipid Lowering Arm (ASCOT-LLA) and the Collaborative Atorvastatin Diabetes Study (CARDS). The studies demonstrated mean reductions with $10 \mathrm{mg}$ of atorvastatin daily of $29 \%$ and $40 \%$ in LDL-C levels, respectively. ASCOT-LLA examined almost 20,000 patients with hypertension and found $29 \%$ less coronary events, albeit no significant reductions of cardiac mortality, with atorvastatin use (Sever et al 2003). CARDS focused on patient with non-insulin-dependent diabetes mellitus and demonstrated a 36\% decrease in coronary events (Colhoun et al 2004). Of note, the ASPEN (Atorvastatin Study for Prevention of coronary heart disease Endpoints in Non-insulin-dependent diabetes mellitus) study showed that despite a $29 \%$ reduction in LDL-C levels ( $\mathrm{p}<0.0001$ compared to placebo), a statistically significant reduction in the primary endpoint of major cardiovascular events (cardiovascular mortality, non-fatal major cardiovascular event or stroke, and unstable angina requiring hospitalization) was not observed with atorvastatin compared to placebo $(13.7 \%$ vs $15.0 \%$, p-value $=\mathrm{NS})$ (Arca 2007). Thus, atorvastatin and simvastatin offer similar benefits as the first generation of statins when titrated for equivalent reductions in LDL-C levels.

The availability of the more potent second generation of statins compared to those of the first generation in the drug class has offered the opportunity to study whether intensive statin therapy - intended to maximize LDL-C reduction - yields even better cardiovascular outcomes than a moderate level of statin therapy, for example those regimens commonly used in the 1990s and early 2000s. Most investigation comparing intensive vs moderate statin therapy has focused on secondary prevention patients, who have been considered most likely to benefit from aggressive lipid-lowering therapy. In the Treating to New Targets (TNT) study, patients with stable coronary artery disease who received $80 \mathrm{mg}$ of atorvastatin daily experienced a $20 \%$ reduction in coronary events (but no reduction in mortality) compared to patients who received $10 \mathrm{mg}$ of atorvastatin daily (LaRosa et al 2005). A similar trial, the Incremental Decrease in End Points Through Aggressive Lipid Lowering (IDEAL) study, compared $80 \mathrm{mg}$ of atorvastatin daily (intensive) and 20-40 mg of simvastatin daily (moderate) in patients with a history of MI, and although it did not find a statistically significant reduction in coronary events with intensive therapy, there was significant reduction of secondary endpoints including coronary events plus stroke, as well as non-fatal MI (Pedersen et al 2005).

The data in support of intensive statin therapy is suggestive of benefit in patients who have recently suffered coronary events. The Myocardial Ischemia Reduction with Aggressive Cholesterol Lowering (MIRACL) study established the utility of high-dose atorvastatin therapy - $80 \mathrm{mg}$ daily - started in patients after acute coronary syndromes. Patients receiving atorvastatin for 16 weeks experienced a $16 \%$ reduction in recurrent coronary events, although no significant effect on mortality was observed (Schwartz et al 2001). The Pravastatin or Atorvastatin Evaluation and Infection Therapy - Thrombolysis in Myocardial Infarction 22 (PROVE IT-TIMI 22) study directly compared regimens comprising $80 \mathrm{mg}$ of atorvastatin daily (intensive) vs $40 \mathrm{mg}$ of pravastatin daily given after acute coronary syndromes (Cannon et al 2004). In those patients who had not previously been on statin therapy, there were reductions in LDL-C levels of $22 \%$ and $51 \%$ with pravastatin and atorvastatin, respectively. Comparing the two 
groups, there was a $16 \%$ reduction in the primary composite end point of CHD death, non-fatal MI, resuscitated sudden cardiac death, or emergency rehospitalization for recurrent ischemia observed in the intensive-therapy group relative to the moderate-therapy group after 2 years; event rates began to diverge as early as 3 months after the start of therapy (Cannon et al 2004). This important finding highlights the need to treat very-high-risk patients to lower LDL-C goals $(<70 \mathrm{mg} / \mathrm{dL})$ than had previously been recommended $(<100 \mathrm{mg} / \mathrm{dL})$ (Grundy et al 2004).

In March 2007, atorvastatin was approved as the first cholesterol-lowering drug to reduce the risk of hospitalization for heart failure. The decision was based on a sub-analysis of the TNT study, which showed a statistically significant reduction in heart failure hospitalization in patients with a prior history of heart failure receiving either $80 \mathrm{mg}(10.6 \%)$ or $10 \mathrm{mg}(17.3 \%$; HR: $0.59,95 \% \mathrm{CI}, \mathrm{p}=0.008)$ of atorvastatin. These findings suggest potential benefit of statins at the level of cardiac myocytes and peripheral vasculature. In this study, for each $1 \mathrm{mg} / \mathrm{dL}$ reduction in LDL-C, the risk of heart failure admission decreased by $0.6 \%(p=0.007)$ (Khush et al 2007).

\section{Third generation statin}

Finally, there is a single commercially available drug in the third, high-potency generation of statins, rosuvastatin. Three unique chemical characteristics of rosuvastatin provide enhanced potency against HMG-CoA reductase. First, in addition to the dihydroxyheptenoic acid side chain common to all statins, rosuvastatin has a fluorinated phenyl group and a polar methane sulphonamide group providing multiple sites of activity against HMG-CoA reductase (Istvan and Desienhofer 2001). Second, atorvastatin and rosuvastatin have enhanced binding enthalpies for HMGCoA reductase. Binding enthalpy refers to the strength of the interaction between the inhibitor and target enzyme. These two statins are the only members of their class to form a hydrogen bond with the hydroxyl group of Ser565 on HMG-CoA reductase. A shorter hydrogen bond length and involvement of sulfonyl groups contribute to superior enzyme inhibition by rosuvastatin (Carbonell and Freire 2005). Third, the hydrophilic nature of rosuvastatin may also reduce the number of drug-drug interactions by eliminating dependence on metabolic conversion to a water-soluble molecule (Holdgate et al 2003).

Rosuvastatin's clinical efficacy has been extensively studied in over 20,000 patients with renal dysfunction $(53 \%)$, hypertension $(52 \%)$, cardiovascular disease $(36 \%)$, diabetes mellitus (17\%), and age greater than 65 years (31\%) (Brewer 2003). In a multicenter, double-blind, placebo-controlled trial, $10 \mathrm{mg}$ rosuvastatin produced reductions in total cholesterol (TC) (35\%), LDL-C (50\%), non-HDL-C/HDL-C (50\%), Apo-B (40\%), and triglyceride (TG) (19\%) with an increase in HDL-C (8\%) within 12 weeks of initiating therapy (Olsson et al 2002). In another study of 129 patients receiving rosuvastatin $(10-40 \mathrm{mg}), \mathrm{LDL}-\mathrm{C}$ was reduced by $47 \%-57 \%$ at 6 weeks vs $38 \%-54 \%$ in patients treated with atorvastatin (10-80 mg) (Schneck et al 2003). The maximum recommended dose of rosuvastatin is $40 \mathrm{mg}$ daily. In an effort to investigate the impact of rosuvastatin on cardiovascular risk reduction, AstraZeneca has embarked on a comprehensive, global program known as GALAXY. The GALAXY program focuses on: 1) atherogenic lipid profiles, 2) changes in atheroma volume, and 3) cardiovascular morbidity and mortality. A number of additional studies focus on specialized populations including diabetics, and ethnic minorities (Schuster 2007).

One of the first studies establishing clinical efficacy and superiority of rosuvastatin over other commonly used statin agents was The Statin Therapies for Elevated Lipid Levels Compared Across Doses to Rosuvastatin (STELLAR) study (Jones et al 2003). A 6-week, parallelgroup, open-label, randomized, multi-center trial in over 2400 patients with hypercholesterolemia, STELLAR compared rosuvastatin $(10-40 \mathrm{mg})$ with atorvastatin $(10-80 \mathrm{mg})$, simvastatin (10-80 mg), and pravastatin (10-40 mg) across clinically applicable dose ranges. Rosuvastatin $(n=473)$ reduced LDL-C by $46 \%-55 \%$ vs $37 \%-51 \%$ with atorvastatin $(\mathrm{n}=641), 28 \%-46 \%$ with simvastatin $(\mathrm{n}=648)$, and $20 \%-30 \%$ with pravastatin $(\mathrm{n}=485)$. Across the range of doses, rosuvastatin reduced LDL-C by a mean of $8.2 \%$ more than atorvastatin, 26\% more than pravastatin, and 12\%-18\% more than simvastatin $(\mathrm{p}<0.001)$. Milligram-equivalent LDL-C reduction was greater with rosuvastatin than all other statins $(\mathrm{p}<0.002)$. Rosuvastatin also reduced TC more significantly than all other statins $(\mathrm{p}<0.001)$ and decreased TG more significantly $(\mathrm{p}<0.001)$ than simvastatin and pravastatin. Similarly, rosuvastatin increased HDL-C by a mean of $7.7 \%-9.6 \%$ compared to $2.1 \%-6.8 \%$ in all other groups.

Two studies have evaluated efficacy of rosuvastatin therapy at 52 weeks. In the first randomized, double-blind, multi-center study, 412 patients with LDL-C ranging between $160-250 \mathrm{mg} / \mathrm{dL}$ received fixed doses of rosuvastatin $(5 \mathrm{mg}$, $\mathrm{n}=138$ or $10 \mathrm{mg}, \mathrm{n}=134)$ or atorvastatin $(10 \mathrm{mg}, \mathrm{n}=140)$ for 12 weeks followed by dose adjustments up to $80 \mathrm{mg}$ if NCEP/ATP II LDL-C goals were not met. Both doses 
of rosuvastatin resulted in greater LDL-C reductions than atorvastatin at 12 weeks ( $46 \%$ and 50\% respectively vs 39\%, $\mathrm{p}<0.001$ for both groups) and 52 weeks (47\% and 53\% respectively vs $44 \%, \mathrm{p}<0.05$ and $\mathrm{p}<0.001)$. Ninety-eight percent of patients treated with rosuvastatin $(10 \mathrm{mg}$ initial dose) achieved NCEP/ATP-II LDL-C goals compared to $87 \%$ with atorvastatin. Notably, $82 \%$ of patients treated with $10 \mathrm{mg}$ rosuvastatin did not require dose titration to achieve goal LDL-C levels as compared to $59 \%$ of patients receiving atorvastatin (10 mg) (Olsson et al 2002).

In a similarly designed trial, 477 hypercholesterolemic patients received fixed doses of rosuvastatin ( $5 \mathrm{mg}$ or $10 \mathrm{mg}$ ), pravastatin $(20 \mathrm{mg})$, or simvastatin $(20 \mathrm{mg}$ ) for 12 weeks followed by 40 weeks of liberal dose titration up to $80 \mathrm{mg}$ for rosuvastatin and simvastatin and $40 \mathrm{mg}$ for pravastatin. At 12 weeks both doses of rosuvastatin had reduced LDL-C levels (39\% and 47\%, respectively) more significantly than pravastatin $(26.5 \%)$ and simvastatin $(34.6 \%)(\mathrm{p}<0.05)$. After 52 weeks, more rosuvastatin-treated patients achieved their ATP II LDL-C goals (88\% and 87.5\%, respectively) than recipients of pravastatin $(60 \%)$ or simvastatin $(72.5 \%)$ (Brown et al 2002).

A unique aspect of the GALAXY program is the scale and breadth of subpopulations studied. Specific focus groups include: Type II diabetics (Tuomilehto et al 2004), hemodialysis recipients, patients with congestive heart failure (Krum et al 2006; Raina et al 2006) and specific ethnic groups. Three prospective studies evaluating rosuvastatin efficacy in under-represented populations have recently been presented, namely (African American Rosuvastatin Investigation of Efficacy and Safety) (Ferdinand et al 2006), STARSHIP (STudy Assessing RoSuvastatin in HIspanic Population) (Llorett et al 2006), and IRIS (Investigation of Rosuvastatin In South Asian Subjects) (Deedwania et al 2006).

The recently published CORONA (Controlled Rosuvastatin Multinational Trial in Heart Failure) compared clinical outcomes for rosuvastatin $10 \mathrm{mg}$ daily vs placebo specifically in 5011 patients with systolic heart failure of ischemic origin aged 60 years or over with New York Heart Association (NYHA) functional class II or III/IV, and had received optimal medical therapy. After 33 months of follow-up (median), only a small difference in the primary composite endpoint of cardiovascular death, non-fatal MI, or non-fatal stroke between the rosuvastatin and placebo treatment groups $(27.5 \%$ vs $29.3 \%, p=N S)$. A post-hoc analysis showed a lower rate of non-fatal or fatal MI or stroke in patients receiving rosuvastatin compared with placebo $(9.0 \%$ vs $10.6 \%$, $\mathrm{HR}=0.84 ; \mathrm{p}=0.05)$

\section{Changes in atheroma volume}

The established benefit of statin therapy has limited clinical trial design due to concerns over the ethics of placebo-controlled randomization in moderate to high-risk populations. These findings have spurred interest in evaluating the direct effects of lipid-modifying therapy on atheromatous plaque burden using surrogate measures of clinical outcomes such as carotid intima-media thickness (CIMT) and coronary plaque volume. Studies such as the Atherosclerosis Progression in Familial Hypercholesterolemia (ASAP) trial and Arterial Biology for the Investigation of the Treatment Effects of Reducing Cholesterol (ARBITER) trial demonstrate the ability of B-mode ultrasound to non-invasively quantitate changes in carotid plaque with statin therapy. The ASAP trial demonstrated plaque regression from baseline in hypercholesterolemic patients treated with atorvastatin $80 \mathrm{mg}$ compared to simvastatin $40 \mathrm{mg}$ ( $-0.031 \mathrm{~mm}$ vs $0.036 \mathrm{~mm}$, $\mathrm{p}<0.0005$ and $\mathrm{p}<0.0017$, respectively) (Smilde et al 2001). The ARBITER study similarly showed a reduction in CIMT by $0.034 \mathrm{~mm}$ with atorvastatin $80 \mathrm{mg}$ vs $0.025 \mathrm{~mm}$ with pravastatin $40 \mathrm{mg}$ after 12 months $(\mathrm{p}=0.03)$ (Taylor et al 2002). The effect of rosuvastatin on CIMT will be prospectively evaluated in the Measuring Effects on intima media Thickness: an Evaluation Of Rosuvastatin (METEOR) study. Low risk subjects $(\mathrm{n}=840)$ with signs of sub-clinical atherosclerosis will be randomized $(5: 2)$ in this parallelgroup study to rosuvastatin $(40 \mathrm{mg}$ ) or placebo for 104 weeks. The primary endpoint will be the change in CIMT from baseline. Secondary endpoints include the effect of rosuvastatin on lipid profiles and C-reactive protein levels (Crouse et al 2004).

The Outcome of Rosuvastatin treatment on carotid artery atheroma: a magnetic resonance Imaging ObservatioN (ORION) study was a randomized, double-blind trial to assess the effect of rosuvastatin on carotid artery atheroma in asymptomatic patients with hypercholesterolemia. Thirtyfive patients with LDL-C between $100-250 \mathrm{mg} / \mathrm{dL}$ and $16 \%-79 \%$ reduction of the carotid artery diameter assessed by ultrasound or MRI received rosuvastatin $5 \mathrm{mg}(\mathrm{n}=15)$ or $40 \mathrm{mg}(\mathrm{n}=20)$ daily. Of note, 14 patients in the high-dose rosuvastatin group were up-titrated to $80 \mathrm{mg}$ daily then back titrated to $40 \mathrm{mg}$ daily ( $80 \mathrm{mg}$ exposure range, $70-701$ days). LDL-C was reduced from baseline by $39 \%$ and $58 \%$ in the low- and high-dose groups, respectively $(\mathrm{p}<0.001)$. There was no significant difference in the changes of the carotid artery wall volume between the groups. In plaques with a lipid-rich core, high-dose rosuvastatin reduced the percentage of lipid-rich necrotic core at the most diseased section by 
$35.5 \%(\mathrm{p}=0.006)$ with $90 \%$ of plaques showing regression from baseline (Hatsukami et al 2005).

Recently, the Measuring Effects on Intima-Media Thickness: an Evaluation of Rosuvastatin (METEOR) was published. In this randomized, double-blind, placebo-controlled study of 948 individuals with minimal coronary risk factors, patients received either rosuvastatin ( $40 \mathrm{mg}$ daily) or placebo. At 2 years follow up, the change in maximum CIMT for the 12 carotid sites was -0.0014 (95\% CI, -0.0041 to 0.0014$)$ $\mathrm{mm} /$ year for the rosuvastatin group vs 0.0131 (95\% CI, $0.0087-0.0174) \mathrm{mm} /$ year for the placebo group $(\mathrm{p}<0.001)$. The change in mean CIMT for the rosuvastatin group for the common carotid artery sites was 0.0004 (95\% CI, -0.0011 to 0.0019$) \mathrm{mm} /$ year $(\mathrm{p}<0.001)$. Taken together, these data suggest that rosuvastatin may reduce progression of atherosclerotic disease, yet fails to induce significant regression (Crouse 2007).

While CIMT provides a surrogate marker of coronary atherosclerosis, volumetric intravascular ultrasound (IVUS) has emerged as a direct measure of changes in coronary plaque burden. Using a $30-40 \mathrm{MHz}$ transducer attached to a catheter, axial resolution of 80-100 microns and lateral resolution of 200-250 microns can be achieved. IVUS was used to evaluate clinical atheromatous progression in the Reversing Atherosclerosis with Aggressive Lipid Lowering (REVERSAL) trial, which compared treatment with 40 $\mathrm{mg}$ pravastatin vs $80 \mathrm{mg}$ atorvastatin in 2163 patients with a mean baseline serum LDL-C level of $150 \mathrm{mg} / \mathrm{dL}$ (Nissen et al 2004). Pravastatin reduced serum LDL levels to 110 $\mathrm{mg} / \mathrm{dL}$, while intensive therapy with atorvastatin reduced LDL-C levels to $79 \mathrm{mg} / \mathrm{dL}$. Notably, levels of high-sensitivity C-reactive protein (hs-CRP) were significantly reduced by $5.2 \%$ with pravastatin therapy vs $36.4 \%$ with atorvastatin therapy. IVUS examination at baseline and at 18 months follow up was performed to evaluate for percent change in atheroma volume. In the pravastatin cohort, $2.7 \%$ progression of atheroma volume was observed vs no significant change in the atorvastatin arm $(-0.4 \%)$. The REVERSAL trial marked the advent of IVUS examination as an effective marker of plaque regression in response to lipid-lowering therapy.

Recently, A Study To Evaluate the Effect of Rosuvastatin On Intravascular Ultrasound-Derived Coronary Atheroma Burden (ASTEROID) demonstrated significant reductions in mean LDL-C (130.4 $\pm 34.3 \mathrm{mg} / \mathrm{dL}$ to $60.8 \pm 20.0 \mathrm{mg} / \mathrm{dL}$, $\mathrm{p}<0.001)$ and increases in mean HDL-C from baseline $(43.1 \pm$ $11.1 \mathrm{mg} / \mathrm{dL}$ to $49.0 \pm 12.6 \mathrm{mg} / \mathrm{dL}, \mathrm{p}<0.001)$ in 349 patients with angiographic evidence of coronary atherosclerosis receiving $40 \mathrm{mg}$ of rosuvastatin daily for 24 months (Nissen et al
2006). IVUS assessment showed reductions in median percent atheroma volume (PAV) from baseline $(-0.79 \% ; 97.5 \% \mathrm{CI}$, $-1.21 \%$ to $-0.53 \%, \mathrm{p}<0.001)$ and median total atheroma volume of $-12.5 \mathrm{~mm}^{3}$ from baseline $(95 \% \mathrm{CI},-15.1$ to -10.5 $\left.\mathrm{mm}^{3}, \mathrm{p}<0.001\right)$. In this prospective, open-label, multi-center, blind endpoints trial PAV was calculated as the percent change in luminal cross-sectional area subtracted from the external elastic membrane cross-sectional area.

While promising, the results of this study were tempered by: 1) the lack of a control group receiving a somewhat less intensive LDL-C lowering regimen, 2) the absence of paired IVUS measurements in less diseased coronary segments demonstrating reproducibility of atheroma volume measurements, and 3) exclusion of patients with coronary stenoses measuring $>50 \%$ throughout a target segment (Blumenthal et al 2006). The clinical correlate to changes in atheroma burden identified by ASTEROID will be evaluated in the outcomes component of GALAXY via studies such as the Justification for the Use of Statins in Primary Prevention: An Intervention Trial Evaluating Rosuvastatin (JUPITER) study (Ridker et al 2003).

\section{Managing statins in clinical practice}

Since 2004, published guidelines have emphasized the need for aggressive LDL-C reduction, resulting in a trend towards more frequent and higher doses of statin therapy in patients. In the era of 'lower is better', the safety of aggressive statin therapy and the effects of lower LDL-C reductions have been raised. Numerous studies support the concept that statin therapy is not merely safe, but beneficial. A meta-anlaysis performed by the Cholesterol Treatment Trialists' (CTT) Collaborators in 2005 evaluated over 90,000 participants in 14 statin trials and demonstrated no significant differences in the rates of death from non-vascular causes in statin treatment groups compared with placebo (3.8\% statin treated vs 4.0 placebo control, $\mathrm{p}=0.1$ ) (Baigent et al 2005). Focusing on 27,000 patients from 5 trials using 'intensive statin therapy', no evidence of any serious adverse effect was associated with low LDL-C levels (Armitage 2007). A recent analysis of the TNT study evaluated the incidence of treatment-associated adverse event rates across quintiles of LDL-C reduction (LaRosa et al 2007). No significant difference in the incidence of treatment associated muscle side effects, changes in liver enzymes, cancer death, hemorrhagic stroke, or suicide were observed across quintiles.

In a recent study, Alsheikh-Ali and colleagues investigated the relationship between low LDL-C levels achieved by statin therapy and adverse effects such as elevated liver 
enzymes, rhabdomyolysis, and cancer (Alsheikh-Ali et al 2007). In this meta-analysis of 23 statin trials with over 309,000 person-years, no significant relationship between percent LDL-C lowering and elevated liver enzymes $\left(\mathrm{R}^{2}<0.001, \mathrm{p}=0.91\right)$, rhabdomyolysis $\left(\mathrm{R}^{2}=0.05, \mathrm{p}=0.16\right)$, or rates of cancer $\left(\mathrm{R}^{2}=0.09, \mathrm{p}=0.92\right)$ were observed. Significantly higher rate of elevated liver enzymes were noted in the high-dose statin recipients compared with the intermediate or low-dose statins treatment groups ( $p<0.001$ for all pair-wise comparisons). The dose-effect was independent of the particular statin used. For each $10 \%$ reduction in LDL-C the rate of elevated liver enzymes was 2.5 times higher with $80 \mathrm{mg}$ of lovastatin, 1.6 times higher with $80 \mathrm{mg}$ of simvastatin, and 4.0 times higher with $80 \mathrm{mg}$ of atorvastatin as compared to their respective low-dose counterparts. Taken together, these data suggest the degree of LDL-C lowering does not correlate with adverse events; rather the dose of statin used may be more relevant. The National Lipid Association (NLA) Statin
Safety Task Force (McKenney et al 2006) has published guidelines regarding the management of statin-associated adverse effects (Table 2).

\section{Muscle toxicity}

Myopathy is defined by any muscular discomfort such as pain, soreness, weakness, or cramping accompanied by an increase in creatine kinase $(\mathrm{CK})$ levels greater than ten times the upper limit of normal. The National Lipid Association Muscle Expert Panel recenty defined 'clinically important rhabdomyolysis as any evidence of muscle cell destruction or enzyme leakage, regardless of the CK level when measured, considered to be causally related to a change in renal function'. The panel suggested the term rhabdomyolysis be replaced by classes of absolute CK elevation with mild CK increases being less than 10 times the upper limit of normal (ULN), moderate increases being greater than or equal to 10 times the ULN, and marked increases being greater than or equal to 50 times the ULN (Thompson et al 2006).

Table 2 Summary of National Lipid Association Statin Safety Recommendations

\section{Muscle effects}

I. Pretreatment measurement of CK levels is generally not necessary unless an individual is at high risk. ${ }^{\mathrm{a}}$

2. Routine measurements of CK levels are unnecessary in asymptomatic patients.

3. Counsel patients on the possiblity of muscle discomfort while on statin therapy and the importance of reporting symptoms.

4. In symptomatic patients, CK levels should be measured.

a. If CK levels $<10$ times the ULN then statin therapy may be continued or doses reduced with close monitoring of symptoms.

b. If CK levels $>10,000 \mathrm{IU} / \mathrm{L}$ or above 10 times the ULN, then admit for IV hydration therapy, monitoring of renal function, and treatment of rhabdomyolysis.

c. Irrespective of CK levels, if muscle symptoms are intolerable, statin therapy should be discontinued with possible reinstitution of a different agent or lower dose once asymptomatic.

d. If symptoms recur, alternative therapies should be considered.

Hepatic effects

I. Measure transaminase levels before initiating therapy, 12 weeks after starting therapy, after a dose adjustment, and periodically thereafter.

2. Monitor for signs of potential hepatotoxicity such as jaundice, malaise, fatigue, and lethargy. If present, measure transaminase levels, fractionated bilirubin levels, and liver function tests.

3. If asymptomatic transaminase levels are between I to 3 times the ULN, then consider continuing statin therapy with close follow up testing.

4. If transaminase levels increase $>3$ times the ULN, then reduce the statin dose or discontinue treatment while ruling out other possible etiologies.

Renal effects

5. If objective evidence of liver injury is documented, then discontinue the statin and refer the patient to a gastroenterologist.

I. Routine measurements of serum creatinine and proetinuria are not necessary for patients on statins.

2. Pre-treatment baseline creatinine levels may be helpful in identifying patients with underlying renal disease who may be at risk for higher muscle toxicity.

3. If creatinine levels increase while on statin therapy, an adjustment in statin dosing may be required.

4. If proteinuria is detected, consider adjusting the statin dose.

5. Any perturbation of renal indices should warrant further investigation of other non-statin related causes.

6. In patients with chronic kidney disease, statin therapy may be intiated with close attention to dose adjustments in moderate to severe renal disease.

Notes: aRisk factors for muscle toxicity include: concomitant therapy with fibric acid derivatives, erythromycin, or azole antifungals, advanced age, small body habitus, worsening renal function, ongoing infection, trauma such as recent surgery, alcohol abuse, and untreated hypothyroidism.

Abbreviations: CK, creatine kinase; ULN, upper limit of normal. 
The most common side effect of statins remains myalgia without changes in CK levels (Bays 2006). In clinical trials, the rates of reported myalgias range between $1.5 \%$ and $3.0 \%$. In clinical practice, the incidence of myalgias is between $0.3 \%$ and $33 \%$, suggesting that selection critera for clinical trials may exclude patients susceptible to statin-induced myalgias.

Law and colleagues reviewed 21 statin-based clinical trials with over 180,000 person years for evidence of muscle toxicity. The incidence of myopathy in this study was 11 per 100,000 person-years. The incidence of rhabdomyolysis in 2 cohort studies was 3.4 (1.6-6.5) per 100,000 person-years and 10 -fold higher when gemfibrozil was used in combination with statins. For statins metabolized by cytochrome P450 3A4 (CYP3A4), such as lovastatin, atorvastatin, and simvastatin), the incidence was 4.2 per 100,000 person-years. In this group, interaction with drugs known to inhibit CYP3A4 (ie, erythromycin and azole antifungals) occurred in $60 \%$ of case (Law and Rudnicka 2006). These findings are supported by reports from the FDA AER's database of 0.3-2.2 cases of myopathy and $0.3-13.5$ cases of rhabdomyolysis per million statin prescriptions (McKenney et al 2006).

The risk of myopathy can be associated with all statins (Davidson et al 2006). In 3 large trials using pravastatin $40 \mathrm{mg}$ daily compared to placebo, there were no reported cases of myopathy. In two trials of atorvastatin $(10 \mathrm{mg}$ daily) vs placebo, three cases of mypathy were documented (ASCOT-LLA and CARDS). The incidence of myopathy was $<0.01 \%$ per year in several studies using simvastatin (20-40 mg daily). No cases of myopathy were reported in patients receiving fluvastatin ( $40 \mathrm{mg}$ twice daily) compared to placebo (Armitage 2007). In a separate study, one patient with renal transplantation and severe trauma experienced rhabdomyolysis (Serruys et al 2002). Lovastatin (40-80 mg daily) is rarely associated with myopathy. Six documented cases were reported in a large trial of 8000 individuals (Bradford et al 1994). In general all statins can cause myopathy with a risk of progressing to rhabdomyolysis. The risk appears to increase with higher doses.

Risk factors for the development of statin-induced myopathy include drug-drug interactions (ie, fibric acid derivatives, erythromycin, and azole antifungals), advaced age, small body habitus, worsening renal function, ongoing infection, trauma such as recent surgery, alcohol abuse, and untreated hypothyroidism. Current guidelines recommend ruling out these possible etiologies whenever patients present with muscle symptoms or an increased CK level while on statin therapy. Pretreatment measurement of CK levels is generally not necessary unless an individual is at high risk of developing myopathy with statin therapy. Routine measurements of CK levels are also unnecessary in asymptomatic patients. Counseling patients on the possiblity of muscle discomfort while on statin therapy and the need to report these complaints to their primary physicians is of paramount importance. In symptomatic patients, $\mathrm{CK}$ levels should be measured. If CK levels are less than 10 times the ULN then statin therapy may be continued or doses reduced with close monitoring of symptoms. If CK levels are greater than 10,000 IU/L or above 10 times the ULN, then patients should be admitted for IV hydration therapy, monitoring of renal function, and treatment of rhabdomyolysis. Irrespective of CK levels, if muscle symptoms are intolerable, statin therapy should be discontinued with possible reinstitution of a different agent or lower dose once asymptomatic. If symptoms recur, alternative therapies should be considered (McKenney et al 2006).

\section{Hepatic effects of statin therapy}

Abnormal hepatic transaminase levels (LFT) are recognized as an infrequent occurrence of statin therapy. In particular, the transaminases (alanine and aspartate) seem to increase within the first 6 months of therapy. In the majority of clinical trials, elevated alanine aminotransferase (ALT) levels greater than three times the upper limit of normal on 2 or more measurements have been considered a safety endpoint. Elevated ALT levels occur in $<1 \%$ of patients receiving low to intermediate dose statins and in $2 \%-3 \%$ of patients on high dose therapy ( $80 \mathrm{mg}$ daily) (Cohen et al 2006). In $70 \%$ of cases, transaminase elevations resolve spontaneously even if statin therapy is continued (Bays 2006). According to the NLA Statin Safety Task Force, the incidence of liver function abnormalities was more common in obese patients, diabetics, older individuals, and those receiving multiple medications. The cause of elevated transaminases remains unknown and does not appear to be related to the degree of LDL-C reduction (McKenney et al 2006).

Current guidelines recommend measuring transaminase levels before initiating therapy, 12 weeks after starting therapy, after a dose adjustment, and periodically thereafter. Signs of potential hepatotoxicity such as jaundice, malaise, fatigue, and lethargy should alert physicians to measure transaminase levels and liver function tests. Fractionated bilirubin levels are recommended to rule out hepatic injury. If isolated asymptomatic transaminase levels are increased between one to three times the ULN, then follow up testing should be performed and the statin may be continued. If transaminase levels increase about three times the ULN, then the test ought to be repeated and other etiologies ruled out. 
The physician should consider reducing the dose of statin or discontinuing the medication based on clinical judgement. If objective evidence of liver injury is documented, then discontinue the statin and refer the patient to a gastroenterologist (Cohen et al 2006; McKenney et al 2006).

\section{Renal effects of statin therapy}

According to a recent report by the NLA Statin Safety Task Force, statins do not appear to be associated with renal failure or insufficiency without concomitant rhabdomyolysis (Kasiske et al 2006). The FDA AERS database reports 0.3-0.9 cases per one million statin prescriptions (Davidson et al 2006). In general, routine measurements of serum creatinine and proetinuria are not necessary for patients on statins. Pre-treatment baseline creatinine levels may be helpful in identifying patients with underlying renal disease who may be at risk for higher muscle toxicity on statin therapy. If creatinine levels are noted to increase while on statin therapy, an adjustment in statin dosing may be required. Discontinuation of the drug is generally not necessary. Similarly, if proteinuria is detected, discontinuing statin therapy is not necessary, while dose adjustments may be reasonable. Any perturbation of renal indices should warrant further investigation of other non-statin related causes. In patients with chronic kidney disease, statin therapy may be initiated with close attention to dose adjustments in moderate to severe renal disease (McKenney et al 2006).

\section{Safety profile of rosuvastatin}

The safety of rosuvastatin has been extensively evaluated in the largest pre-marketing clinical development program for any statin currently in use. Over 12,500 patients received rosuvastatin 5-80 mg in 27 controlled clinical trials over 6-96 weeks. Over 5000 patients received the highest marketed dose (40 mg) (Food and Drug Administration 2006). In this analysis, rates of pharyngitis, headache, diarrhea, dyspepsia, nausea, myalgia, and withdrawal rates due to adverse effects were similar to currently marketed statins. Myopathy as defined by creatine kinase $(\mathrm{CK})$ elevation greater than three times the upper limit of normal (ULN) occurred in $0.1 \%-0.4 \%$ of patients receiving 5-40 mg rosuvastatin with an overall rate of $0.2 \%$ for all doses. No cases of rhabdomyolysis were reported (McAfee et al 2006).

Hepatotoxicity as defined as increased alanine transaminase (ALT) by three times the ULN were reported in $0.5 \%, 0.1 \%, 0.1 \%$, and $0.3 \%$ of patients receiving 5,10 , 20 , and $40 \mathrm{mg}$ of rosuvastatin (McAfee et al 2006). Similar results $(0.2 \%)$ have been reported across the dose ranges of atorvastatin, simvastatin, and pravastatin (Schneck et al 2003; Grundy 2005). As a result, serum transaminases should be monitored prior to initiation of statin therapy, 12 weeks following initiation and every 6 months thereafter

Dipstick-positive proteinuria was reported in $0.2 \%, 0.6 \%$, and $0.7 \%$ of patients receiving 5,10 , or $20 \mathrm{mg}$ of rosuvastatin compared to $0.6 \%$ receiving placebo. The incidence of proteinuria was similar to reported rates with atorvastatin, simvastatin, and pravastatin across dose ranges. Urinary protein was predominantly of low molecular weight by gel electrophoresis, suggesting decreased reabsorption vs increased glomerular leakage as the primary cause. Reported proteinuria was transient, reversible, and not associated with a reduction in glomerular filtration rate (GFR) in long-term follow up (McAfee et al 2006). In 2005, a critical analysis of 38 reports of renal insufficiency in patients treated with rosuvastain, led the FDA to conclude that "no consistent pattern of clinical presentation or of renal injury is evident among the cases of renal failure reported to date that clearly indicate causation by [rosuvastatin]' (Zipes et al 2006).

A recent analysis of the Rosuvastatin Clinical Development Program reviewed 3956 patients treated with short-term (6-8 weeks) of rosuvastatin $(5-40 \mathrm{mg})$ to determine the effect of rosuvastatin treatment on the estimated glomerular filtration rate (eGFR). For each dose of rosuvastatin, eGFR significantly increased individually and for all doses combined vs baseline (range +0.9 to $+3.2 \mathrm{~mL} / \mathrm{min} / 1.73 \mathrm{~m}^{2}$ ). In 525 patients from placebo-controlled trials rosuvastatin increased eGFR by $+0.8 \mathrm{~mL} / \mathrm{min} / 1.73 \mathrm{~m}^{2}(95 \% \mathrm{CI}$ +0.1 to +1.5$)$ compared to baseline $(\mathrm{p}<0.04)$ and placebotreated patients $(95 \% \mathrm{CI}-2.5$ to $-0.5, \mathrm{p}<0.001)$ (Vidt et al 2006). These data suggest a potential beneficial effect of 'short-term' rosuvastatin on renal function.

Since FDA approval in 2003, few studies have evaluated the safety of rosuvastatin in actual clinical practice. A Dutch historical cohort study in over 45,000 patients compared the incidence of adverse event reports (AER: myopathy, rhabdomyolysis, acute renal failure, and hepatic impairment) in those receiving rosuvastatin $(n=10,147)$ with those receiving other statins $(n=37,396)$ and statin-naïve $(n=99,935)$ individuals from 2003 to 2004 . In this analysis, the incidence of AER was lower than 1 in 3000 person-years' exposure to statins, with no significant difference between recipients of rosuvastatin compared to other statins. The incidence per 1000 person-years for patients treated with rosuvastatin was $0.00,0.00,0.31,0.00$, and 5.34 for myopathy, rhabdomyolysis, acute renal failure, hepatic impairment, and allcause mortality, respectively. In the 'other-statins' cohort, 
the incidence per 1000 person-years was $0.03,0.00,0.20$, 0.11 , and 11.48 for myopathy, rhabdomyolysis, renal failure, hepatic impairment, and all-cause mortality, respectively (Goettsch et al 2006).

An American post-marketing analysis from August 2003 through October 2004 reported 26 cases of myopathy and rhabdomyolysis yielding a 1-year myopathy/rhabdomyolysis incidence of 0.43 per 100,000 rosuvastatin prescriptions (Zipes et al 2006). Another post-marketing analysis compared AERs for rosuvastatin vs simvastatin, pravastatin, and atorvastatin between October 2003 and September 2004. In the concurrent time period, the composite rate of rhabdomyolysis, proteinuria/nephropathy, or renal failure was higher in those treated with rosuvastatin compared to all other statins analyzed ( $p<0.001$ per group) (Al-sheikh-Ali et al 2005).

\section{Real-world effectiveness: implications for patient management}

Substantial evidence suggests that dyslipidemia is undertreated in real-world clinical practice. Before publication of the NCEP-ATPIII guidelines in 2001, an analysis of 4888 patients with dyslipidemia from 5 regions across the United States revealed that only 38\% achieved specified NCEP LDL-C targets. Especially concerning was the finding that among patients with established CHD, only $18 \%$ achieved LDL-C targets (Pearson et al 2000). In 2005, a national survey of 4885 patients demonstrated some improvement in attainment of LDL-C goals, however patients with diabetes (55\%) and other CHD risk equivalents (40\%) were less likely to have achieved their LDL cholesterol targets than those with CHD (62\%) (Davidson et al 2005). Suggested mechanisms for poor results in real-world practice include: failure to identify candidates for pharmacologic therapy, failure to appropriately titrate drug therapy, poor patient adherence and follow-up, and cost-related issues (Davidson 2006).

Few studies focus on the effectiveness of statin-based treatment for dyslipidemia. An analysis of 8251 patients in a managed care database receiving treatment with rosuvastatin, atorvastatin, simvastatin, pravastatin, lovastatin, and fluvastatin between 2003 and 2004 demonstrated greater absolute and percent reductions in LDL-C, TG, and TC levels with rosuvastatin than with other statins (all $\mathrm{p}<0.05$ except for TG reduction vs atorvastatin). In this study, NCEP-ATP III goal attainment was higher with rosuvastatin than with other statins after adjustment for age, sex, baseline LDL, risk status, dose, and duration of therapy ( $\mathrm{p}<0.05$ ) (Bullano et al 2006). Another study evaluated 775 high-risk patients (defined as having CHD or CHD risk-equivalents) from 500 physician offices in the mid-western US receiving statin treatment. In this analysis, percent LDL-C reduction was significantly greater with rosuvastatin vs atorvastatin or simvastatin $(37 \%$ vs $28 \%$ or $27 \%$, respectively, $\mathrm{p}<0.05)$. The estimated percentage of patients achieving target goals set for by the NCEP-ATPIII was significantly higher $(\mathrm{p}<0.05)$ with rosuvastatin $(69.7 \%)$ compared with atorvastatin $(54.8 \%)$ or simvastatin $(51.2 \%)$ after adjusting for baseline characteristics (Ohsfeldt et al 2006).

For those patients in whom statin monotherapy is insufficient to reach the desired LDL-C goal as laid out by the NCEP ATP III guidelines, an attractive adjunctive pharmacological option has emerged with the introduction of ezetimibe, the first of a novel class of cholesterol drugs that works by inhibiting uptake of cholesterol in the small intestine. It therefore exploits a different mechanism than the statin drugs, with the potential for synergy between the two classes. The addition of ezetimibe to atorvastatin or to simvastatin can result in an additional 10\%-20\% reduction in LDL-C levels (Bays et al 2004; Stein et al 2004). The recently published EXPLORER study investigated the efficacy and safety of rosuvastatin (40 mg) alone or in combination with ezetimibe $(10 \mathrm{mg})$ in 469 patients at high risk of CHD. After 6 weeks of therapy, significantly more patients receiving rosuvastatin/ezetimibe than rosuvastatin alone achieved ATP III LDL-C cholesterol goals $(<100 \mathrm{mg} / \mathrm{dL}$, $94.0 \%$ vs $79.1 \%, \mathrm{p}<0.001)$ and the optional LDL-Cl goal $(<70 \mathrm{mg} / \mathrm{dL})$ for very high-risk patients $(79.6 \%$ vs $35.0 \%$, $\mathrm{p}<0.001)$. The combination of rosuvastatin/ezetimibe reduced LDL-C significantly more than rosuvastatin $(69.8 \%$ vs $57.1 \%$, p < 0.001) (Ballantyne et al 2007). While it is not yet clear whether this enhanced lipid reduction will translate into improved cardiovascular outcomes, a meta-analysis of statin and non-statin therapies showed similar improvement in CHD risk that correlated with levels of LDL-C reduction (Robinson et al 2005). Controlled clinical trials comparing outcomes with a combination of a statin and ezetimibe against statin monotherapy are underway.

\section{Future directions}

Statins have emerged as the global leader in pharmacologic therapy for dyslipidemia. As evidence supporting aggressive reductions in LDL-C continues to grow, the use of statins, especially second and third generation drugs, will continue to grow in parallel. The third generation statin, rosuvastatin, has demonstrated reasonable clinical efficacy and safety in several clinical trials and post-marketing analyses. 
Safety issues surrounding the use of high-potency statins remain of paramount concern. Recent studies support the concept that the degree of LDL-C reduction does not correlate with adverse events, but rather appears to be a dose-dependent phenomenon. A clear understanding of the guidelines regarding statin safety will be essential for physicians managing patients with dyslipidemia.

In addition to reducing LDL-C levels, an emerging target of therapy is high-density lipoprotein cholesterol (HDL-C). A recent analysis of the TNT study demonstrated that in subjects with LDL-C below $70 \mathrm{mg} / \mathrm{dL}$, individuals in the highest quintile of HDL-C levels were are significantly lower risk for major cardiovascular events as compared to those in the lowest HDL-C quintile $(\mathrm{p}<0.03)$ (Barter et al 2007). These data suggest that in individuals achieving LDL-C goals with statin therapy, HDL-C may be an important target of therapy.

The effect of various agents on HDL-C was evaluated in the Comparative Effects on Lipid Levels (COMPELL) trial (Jones 2006). This study determined the relative efficacy of combination therapy with a statin and niacin or ezetimibe compared with a statin alone over 12 weeks. Coadministration of niacin (500 mg up-titrated to $2000 \mathrm{mg}$ ) with atorvastatin (20-40 mg, $\mathrm{n}=60)$ or rosuvastatin $(10-20 \mathrm{mg}, \mathrm{n}=65)$ decreased LDL-C by $56 \%$ and $51 \%$ and increased HDL-C by $22 \%$ and $24 \%$, respectively ( $p=N S$ ). While simvastatin plus ezetimibe decreased LDL-C by $57 \%$, HDL-C only increased by $10 \%$ compared to baseline. Rosuvastatin $(10-40 \mathrm{mg})$ monotherapy decreased LDL-C by $53 \%$ and raised HDL-C by $7 \%$. Future studies involving rosuvastatin/fenofibrate combination therapy and the recently announced combination of rosuvastatin with a next generation fenofibrate (ABT-335) will provide further insight into the efficacy of dual-targeted therapy on both LDL-C and HDL-C profiles.

\section{References}

Alsheikh-Ali AA, Ambrose MS, Kuvin JT, et al. 2005. The safety of rosuvastatin as used in common clinical practice: a postmarketing analysis. Circulation, 111:3051-7.

Alsheikh-Ali A, Maddukuri PV, Han H, et al. 2007. Effect of the magnitude of lipid lowering on risk of elevated liver enzymes, rhabdomyolysis, and cancer: insights from large randomized statin trials. J Am Coll Cardiol, 50:409-18.

Arca, Marcello. 2007. Atorvastatin efficacy in the prevention of cardiovascular events in patients with diabetes mellitus and/or metabolic syndrome. Drugs, 67:43-54.

Armitage J. 2007. The safety of statins in clinical practice. Lancet. Jun 6; [Epub ahead of print]

[ALLHAT] The ALLHAT Officers and Coordinators for the ALLHAT Collaborative Research Group. 2002. Major outcomes in moderately hypercholesterolemic, hypertensive patients randomized to pravastatin vs usual care. JAMA, 288:2998-3007.

Baigent C, Keech A, Kearney PM, et al. 2005. Efficacy and safety of cholesterollowering treatment: prospective meta-analysis of data from 90,056 participants in 14 randomised trials of statins. Lancet, 366:1267-78.
Ballantyne CM, Weiss R, Moccetti T, et al. 2007. Efficacy and safety of rosuvastatin $40 \mathrm{mg}$ alone or in combination with ezetimibe in patients at high risk of cardiovascular disease (results from the EXPLORER study). Am J Cardiol, 99:673-80.

Barter P, Gotto AM, LaRosa JC, et al. 2007. HDL cholesterol, very low levels of LDL cholesterol, and cardiovascular events. $N$ Engl J Med, 357:1301-10.

Bays H. 2006. Statin safety: an overview and assessment of the data -2005 . Am J Cardiol, 97:6C-26C.

Bays H, Ose L, Fraser N, et al. 2004. A multicenter, randomized, doubleblind, placebo-controlled, factorial design study to evaluate the lipidaltering efficacy and safety profile of the ezetimibe/simvastatin tablet compared with ezetimibe and simvastatin monotherapy in patients with primary hypercholesterolemia. Clin Ther, 26:1758-73.

Blumenthal RS, Kapur NK. 2006. Can a potent statin actually regress coronary atherosclerosis? JAMA, 295:1583-4.

Bradford RH, Shear CL, Chremos AN, et al. 1994. Expanded Clinical Evaluation of Lovastatin (EXCEL) study results: two-year efficacy and safety follow-up. Am J Cardiol, 74:667-73.

Brewer HB. 2003. Benefit-risk assessment of Rosuvastatin 10 to 40 milligrams. Am J Cardiol, 92:23K-29K.

Brown WV, Bays HE, Hassman DR, et al. 2002. Efficacy and safety of rosuvastatin compared with pravastatin and simvastatin in patients with hypercholesterolemia: a randomized, double-blind, 52-week trial. Am Heart J, 144:1036-43.

Bullano MF, Wertz DA, Yang GW, et al. 2006. Effect of rosuvastatin compared with other statins on lipid levels and National Cholesterol Education Program goal attainment for low-density lipoprotein cholesterol in a usual care setting. Pharmacotherapy, 26:469-78.

Cannon CP, Braunwald E, McCabe CH, et al. 2004. Intensive versus moderate lipid lowering with statins after acute coronary syndromes. $N \mathrm{Engl}$ J Med, 350:1495-504.

Carbonell T, Freire E. 2005. Binding thermodynamics of statins to HMGCoA reductase. Biochemistry, 44:11741-48.

Cohen DE, Anania FA, Chalasani N. 2006. An assessment of statin safety by hepatologists. Am J Cardiol, 97(suppl 8A):77C-81C.

Colhoun HM, Betteridge DJ, Durrington PN, et al. 2004. Primary prevention of cardiovascular disease with atorvastatin in type 2 diabetes in the Collaborative Atorvastatin Diabetes Study (CARDS): multicentre randomised placebo-controlled trial. Lancet, 364:685-96.

Crouse JR, Grobbee DE, O'leary DH, et al. 2004. Measuring Effects on intima media Thickness: an Evaluation Of Rosuvastatin in subclinical atherosclerosis - the rationale and methodology of the METEOR study. Cardiovasc Drugs Ther, 18:231-8.

Crouse JR 3rd, Raichlen JS, Riley WA, et al; METEOR Study Group. 2007. Effect of rosuvastatin on progression of carotid intima-media thickness in low-risk individuals with subclinical atherosclerosis: the METEOR Trial. JAMA, 297:1344-53.

Davidson MH. 2006. Differences between clinical trial efficacy and realworld effectiveness. Am J Manag Care, 12(15 Suppl):S405-11.

Davidson MH, Clark JA, Glass LM, et al. 2006. Statin safety: an appraisal from the adverse event reporting system. Am J Cardiol, 97(8A):32C-43C.

Davidson MH, Maki KC, Pearson TA, et al. 2005. Results of the National Cholesterol Education (NCEP) Program Evaluation ProjecT Utilizing Novel E-Technology (NEPTUNE) II survey and implications for treatment under the recent NCEP Writing Group recommendations. Am J Cardiol, 96:556-63.

Davignon J, Laaksonen R. 1999. Low-density lipoprotein-independent effects of statins. Curr Opin Lipidol, 10:543-59.

Deedwania P, Gupta M, Stein M, et al. 2006. First large randomized trial of a statin therapy in south asian patients at risk for coronary heart disease: The IRIS trial. Atheroscler Suppl, 206:161.

Downs JR, Clearfield M, Weis S, et al. 1998. Primary prevention of acute coronary events with lovastatin in men and women with average cholesterol levels: results of AFCAPS/TexCAPS. JAMA, 279:1615-22.

Endo A. 2004. The origin of the statins. Atheroscler Suppl, 5:125-30. 
Expert Panel on Detection, Evaluation, and Treatment of High Blood Cholesterol in Adults. 1993. Summary of the second report of the National Cholesterol Education Program (NCEP) Expert Panel on Detection, Evaluation, and Treatment of High Blood Cholesterol in Adults (Adult Treatment Panel II). JAMA, 269:3015-23.

Expert Panel on Detection, Evaluation, and Treatment of High Blood Cholesterol in Adults. 2001. Executive summary of the third report of the National Cholesterol Education Program (NCEP) Expert Panel on Detection, Evaluation, and Treatment of High Blood Cholesterol in Adults (Adult Treatment Panel III). JAMA, 285:2486-97.

Ferdinand KC, Clark LT, Watson KE, et al. 2006. Comparison of efficacy and safety of rosuvastatin versus atorvastatin in African-American patients in a six-week trial. Am J Cardiol, 97:229-35.

Food and Drug Administration. 2006. FDA advisory committee meeting briefing document NDA 21-366 for the use of Crestor. Accessed July 7, 2006. URL: www.fda.gov/ohrms/dockets/ac/03/briefing/3968B1_02_AFDA-Clinical\%20Review.pdf

Goettsch WG, Heintjes EM, Kastelein JJ, et al. 2006. Results from a rosuvastatin historical cohort study in more than 45000 Dutch statin users, a PHARMO study. Pharmacoepidemiol Drug Saf, 15:435-43.

Grundy SM. 2005. The issue of statin safety: where do we stand? Circulation, 111:3016-19.

Grundy SM, Cleeman JI, Merz CN, et al. 2004. Implications of recent clinical trials for the National Cholesterol Education Program Adult Treatment Panel III Guidelines. J Am Coll Cardiol, 44:720-32.

Hatsukami T, Zhao XQ, Kraiss LW, et al. 2005. Assessment of rosuvastatin treatment on carotid atherosclerosis in moderate hypercholesterolemic subjects using magnetic resonance imaging. Eur Heart J, 26(Abstract suppl):626.

Heart Protection Study Collaborative Group. 2002. MRC/BHF Heart Protection Study of cholesterol lowering with simvastatin in 20536 high-risk individuals: a randomised placebo-controlled trial. Lancet, 360:7-22.

Holdaas H, Fellstrom B, Jardine AG, et al. Effect of fluvastatin on cardiac outcomes in renal transplant recipients: a multicentre, randomised, placebo-controlled trial. 2003. Lancet, 361:2024-31.

Holdgate GA, Ward WH, McTaggert F. 2003. Molecular mechanism for inhibition of 3-hydroxy-3-methylglutaryl CoA (HMG-CoA) reductase by rosuvastatin. Biochem Soc Trans, 31):528-31.

Istvan ES, Desienhofer J. 2001. Structural mechanism for statin inhibition of HMG-CoA reductase. Science, 292:1160-4.

Jones P. 2006 International Symposium on Atherosclerosis; June 18-22, 2006; Rome, Italy.

Jones P, Kafonek S, Laurora I, et al. 1998. Comparative dose efficacy study of atorvastatin versus simvastatin, pravastatin, lovastatin, and fluvastatin in patients with hypercholesterolemia (the CURVES study). Am J Cardiol, 81:582-7.

Jones PH, Davidson MH, Stein EA, et al. 2003. Comparison of the efficacy and safety of rosuvastatin versus atorvastatin, simvastatin, and pravastatin across doses (STELLAR* Trial). Am J Cardiol, 92:152-60.

Kasiske BL, Wanner C, O’Neill WC. 2006. An assessment of statin safety by nephrologists. Am J Cardiol, 97(suppl 8A):82C-85C.

Khush KK, Waters DD, Bittner V, et al. 2007. Effect of high-dose atorvastatin on hospitalizations for heart failure: subgroup analysis of the Treating to New Targets (TNT) study. Circulation, 115:576-83.

Krum H, Tonkin A, et al. 2006. The Rosuvastatin Impact on Ventricular Remodelling Cytokines and Neurohormones (UNIVERSE) study [Abstract]. J Am Coll Cardiol, 26:61A-62A.

LaRosa JC, Grundy SM, Kastelein JJ, et al. 2007. Safety and efficacy of atorvastatin-induced very low-density lipoprotein cholesterol levels in patients with coronary heart disease (a post hoc analysis of the treating to new targets [TNT] study). Am J Cardiol, 100:747-52.

LaRosa JC, Grundy SM, Waters DD, et al. 2005. Intensive lipid lowering with atorvastatin in patients with stable coronary disease. $N$ Engl $J$ Med, 352:1425-35.

Law M, Rudnicka AR. 2006. Statin safety: a systematic review. Am $J$ Cardiol, 97:52C-60C.
[LIPID] The Long-Term Intervention with Pravastatin in Ischaemic Disease (LIPID) Study Group. 1998. Prevention of cardiovascular events and death with pravastatin in patients with coronary heart disease and a broad range of initial cholesterol levels. N Engl J Med, 339:1349-57.

Llorett R, Haffner S, Ycas J, Stein M, et al; for the STARSHIP Study Group. 2006. Lipid-lowering results from the first large-scale trial of statin therap in Hispanic-American patients with hypercholesterolemia: STARSHIP [Abstract]. J Am Coll Cardiol, 26:349A-50A.

McAfee AT, Ming EE, Seeger JD, et al. 2006. The comparative safety of rosuvastatin: a retrospective matched cohort study in over 48,000 initiators of statin therapy. Pharmacoepidemiol Drug Saf, 15:444-53.

McKenney JM, Davidson MH, Jacobson TA, et al. 2006. Final conclusions and recommendations of the National Lipid Association Statin Safety Assessment Task Force. Am J Cardiol, 97:89C-94C.

Nissen SE, Nicholls SJ, Sipahi I, et al. 2006. Effect of very high-intensity statin therapy on regression of coronary atherosclerosis: the ASTEROID trial. JAMA, 295:1556-65.

Nissen SE, Tuzcu EM, Schoenhagen P, et al. 2004. Effect of intensive compared with moderate lipid-lowering therapy on progression of coronary atherosclerosis: a randomized controlled trial. JAMA, 291:1071-80.

Ohsfeldt RL, Gandhi SK, Fox KM, et al. 2006. Effectiveness and costeffectiveness of rosuvastatin, atorvastatin, and simvastatin among high-risk patients in usual clinical practice. Am J Manag Care, 12(15 Suppl):S412-23.

Olsson AG, Istad H, Luurila O, et al. 2002. Effects of rosuvastatin and atorvastatin compared over 52 weeks of treatment in patients with hypercholesterolemia. Am Heart J, 144:1044-51.

Pearson TA, Laurora I, Chu H, et al. 2000. The lipid treatment assessment project (L-TAP): a multicenter survey to evaluate the percentages of dyslipidemic patients receiving lipid-lowering therapy and achieving low-density lipoprotein cholesterol goals. Arch Intern Med, 160:459-67.

Pedersen TR, Faergeman O, Kastelein JJ, et al. 2005. High-dose atorvastatin vs usual-dose simvastatin for secondary prevention after myocardial infarction: the IDEAL study: a randomized controlled trial. JAMA, 294:2437-45.

Raina A, Pickering T, Shimbo D. 2006. Statin use in heart failure - A cause for concern? Am Heart J, 152:39-49.

Ridker PM; JUPITER study group. 2003. Rosuvastatin in the primary prevention of cardiovascular disease among patients with low levels of low-density lipoprotein cholesterol and elevated high-sensitivity C-reactive protein: rationale and design of the JUPITER trial. Circulation, 108:2292-7.

Robinson JG, Smith B, Maheshwari N, et al. 2005. Pleiotropic effects of statins: Benefit beyond cholesterol reduction? A meta-regression analysis. J Am Coll Cardiol, 46:1855-62.

Sacks FM, Pfeffer MA, Moye LA, et al. 1996. The effect of pravastatin on coronary events after myocardial infarction in patients with average cholesterol levels. N Engl J Med, 335:1001-9.

Scandinavian Simvastatin Survival Study Group. 1994. Randomised trial of cholesterol lowering in 4444 patients with coronary heart disease: the Scandinavian Simvastatin Survival Study (4S). Lancet, 344:1383-9.

Schneck DW, Knopp RH, Ballantyne CM, et al. 2003. Comparative effects of rosuvastatin and atorvastatin across their dose ranges in patients with hypercholesterolemia and without active arterial disease. $\mathrm{Am} \mathrm{J}$ Cardiol, 91:33-41.

Schwartz GG, Olsson AG, Ezekowitz MD, et al. 2001. Effects of atorvastatin on early recurrent ischemic events in acute coronary syndromes: the MIRACL study - a randomized controlled trial. JAMA, 285:1711-18S.

Schuster H. 2007. The GALAXY Program: an update on studies investigating efficacy and tolerability of rosuvastatin for reducing cardiovascular risk. Investigating cardiovascular risk reduction - the Rosuvastatin GALAXY Programme. Expert Rev Cardiovasc Ther, 5:177-93 
Serruys PW, de Feyter P, Macaya C, et al. 2002. Fluvastatin for prevention of cardiac events following successful first percutaneous coronary intervention: a randomized controlled trial. JAMA, 287:3215-22.

Sever PS, Dahlof B, Poulter NR, et al. 2003. Prevention of coronary and stroke events with atorvastatin in hypertensive patients who have average or lower-than-average cholesterol concentrations, in the Anglo-Scandinavian Cardiac Outcomes Trial-Lipid Lowering Arm (ASCOT-LLA): a multicentre randomised controlled trial. Lancet, 361:1149-58.

Shepherd J, Cobbe SM, Ford I, et al. 1995. Prevention of coronary heart disease with pravastatin in men with hypercholesterolemia. $N$ Engl $J$ Med, 333:1301-7.

Shepherd J, Blauw GJ, Murphy MB, et al. 2002. Pravastatin in elderly individuals at risk of vascular disease (PROSPER): a randomised controlled trial. Lancet, 360:1623-30.

Smilde TJ, van Wissen S, Wollersheim H, et al. 2001. Effect of aggressive versus conventional lipid lowering on atherosclerosis progression in familial hypercholesterolaemia (ASAP): a prospective, randomised, double-blind trial. Lancet, 357:577-81.
Stein E, Stender S, Mata P, et al; for the Ezetimibe Study Group. 2004. Achieving lipoprotein goals in patients at high risk with severe hypercholesterolemia: efficacy and safety of ezetimibe co-administered with atorvastatin. Am Heart J, 148:447-55.

Taylor AJ, Kent SM, Flaherty PJ, et al. 2002. ARBITER: Arterial Biology for the Investigation of the Treatment Effects of Reducing Cholesterol: a randomized trial comparing the effects of atorvastatin and pravastatin on carotid intima medial thickness. Circulation, 106:2055-60.

Thompson PD, Clarkson PM, Rosenson RS, et al. 2006. An assessment of statin safety by muscle experts. Am J Cardiol, 97:69C-76C.

Tuomilehto J, Leiter LA, Kallend D. 2004. A review of the efficacy of rosuvastatin in patients with type 2 diabetes. Int J Clin Pract Suppl, 143:30-40.

Vaughan CJ, Gotto AM Jr. 2004. Update on statins: 2003. Circulation, 110:886-92.

Vidt DG, Harris S, McTaggart F, et al. 2006. Effect of short-term rosuvastatin treatment on estimated glomerular filtration rate. Am J Cardiol, 97:1602-6.

Zipes DP, Zvaifler NJ, Glassock RJ, et al. 2006. Rosuvastatin: an independent analysis of risks and benefits. Med Gen Med, 8:73. 
